\title{
On Relative Orbital Rotation in Relativity Theory $^{1}$
}

\author{
David B. Malament \\ Department of Logic and Philosophy of Science \\ 3151 Social Science Plaza \\ The University of California, Irvine \\ Irvine, CA 92697 \\ dmalamen@uci.edu
}

I want to consider this question within the framework of relativity theory: given two point particles $X$ and $Y$, if $Y$ is rotating relative to $X$, does it follow that $X$ is rotating relative to $\mathrm{Y}$ ? To keep the discussion as simple as possible, I'll allow $\mathrm{X}$ and $\mathrm{Y}$ to be test particles.

As it stands, the question is ambiguous. Roughly speaking, one wants to say that "Y is rotating relative to (or around) $\mathrm{X}$ ", at least in the sense I have in mind, if "the direction of $\mathrm{Y}$ relative to $\mathrm{X}$ " is "changing over time". What must be explained is how to understand the quoted expressions. There is a perfectly straightforward way to do so within Newtonian particle mechanics (section I), where there is an invariant notion of "time", and "space" is assumed to have Euclidean structure. At all times, there is a well-defined vector that points from $\mathrm{X}$ to $\mathrm{Y}$, and one can use it to define the angular velocity of $Y$ relative to $X$.

But the situation is more delicate in relativity theory. Here no such simple interpretation of "relative rotation" is available, and some work is required to make sense of the notion at all. (It seems to me unfortunate that this is often overlooked by parties on both sides when it is debated whether relativity theory supports a "relativist" conception of rotation.) In section II, I'll consider one way of defining the "angular velocity of Y relative to X" (Rosquist (1980)) that does not presuppose the presence of special background spacetime structure (e.g., flatness, asymptotic flatness, stationarity, rotational symmetry), and can be explained in terms of simple (idealized) experimental procedures. I'll also derive an expression for the angular 
velocity of $Y$ relative to $X$ in the special case where the worldlines of $X$ and $Y$ are the images of) integral curves of a common background Killing field. Finally, in section III, I'll turn to the original question.

For purposes of motivation, let's first consider relative rotation within the framework of Newtonian particle mechanics. Here we can associate with the particles, at every time $t$, a relative position vector $\vec{r}_{X Y}(t)$ that gives the position of $Y$ relative to $X$. (We can think of the vector as having its tail coincident with $X$ and its head coincident with $Y$.) The inverted vector, $\vec{r}_{Y X}(t)=-\vec{r}_{X Y}(t)$ gives the position of $\mathrm{X}$ relative to $\mathrm{Y}$ at time $\mathrm{t}$. Let's take for granted that the particles never collide (so that $\vec{r}_{X Y}(t)$ is non-zero at all times), and consider the normalized vector:

$$
\vec{n}_{X Y}(\mathrm{t})=\frac{\vec{r}_{X Y}(\mathrm{t})}{\left|\vec{r}_{X Y}(\mathrm{t})\right|} \text {. }
$$

We can think of it as giving the direction of $Y$ relative to $X$ at time $t$. The (instantaneous) angular velocity of $Y$ relative to $X$ at time $t$ is given by the vector cross product:

$$
\vec{\Omega}_{X Y}(\mathrm{t})=\overrightarrow{\mathrm{n}}_{X Y}(\mathrm{t}) \times \frac{\mathrm{d}}{\mathrm{dt}}\left(\overrightarrow{\mathrm{n}}_{X Y}(\mathrm{t})\right)
$$

Notice that it is not here presupposed that $\mathrm{X}$ is in a state of uniform rectilinear motion. $X$ (and $Y$ too) can wiggle so long as $\vec{n}_{X Y}(t)$ has a well-defined derivative. Notice also that if $\overrightarrow{\mathrm{n}}_{Y X}(\mathrm{t})$ and $\vec{\Omega}_{Y X}(\mathrm{t})$ are defined in the obvious way, by interchanging the roles of $X$ and $Y$, then $\vec{n}_{Y X}(t)=-\vec{n}_{X Y}(t)$ and $\vec{\Omega}_{Y X}(t)=\vec{\Omega}_{X Y}(t)$. We will be interested in two assertions.

(i) $Y$ is not rotating relative to $X$ :

$$
\vec{\Omega}_{X Y}(t)=0 \text { (or, equivalently, } \frac{d}{d t}\left(\vec{n}_{X Y}(t)\right)=0 \text { ) for all } t .
$$


(ii) $Y$ is rotating relative to $X$ with constant angular velocity (i.e., in a fixed plane with constant angular speed): $\frac{\mathrm{d}}{\mathrm{dt}}\left(\vec{\Omega}_{X Y}(\mathrm{t})\right)=\mathbf{0}$ for all $\mathrm{t}$.

What is important for present purposes is that both assertions are manifestly symmetric in $X$ and $Y{ }^{2}$ It is the purpose of the present modest note to show that the situation changes, and changes radically, when one passes to the context of general relativity. We show with an example in section III that there it is possible for $Y$ to be non-rotating relative to $X$, and yet for $X$ to be rotating relative to $Y$ with constant (non-zero) angular velocity. Moreover, the $\mathrm{X}$ and $\mathrm{Y}$ in question can be chosen so that the distance between them is constant (according to any reasonable standard of distance). And the distance can be arbitrarily small. (Of course, it remains to explain the interpretation of relative orbital rotation in general relativity on which these claims rest.) ${ }^{3}$

\section{II}

Let's now turn to the relativity theory. In what follows, let $\left(\mathrm{M}, \mathrm{g}_{\mathrm{ab}}\right)$ be a relativistic spacetime structure, i.e., a pair consisting of a smooth, connected 4manifold $\mathrm{M}$, and a smooth semi-Riemannian metric $\mathrm{g}_{\mathrm{ab}}$ on $\mathrm{M}$ of Lorentz signature $(+1,-1,-1,-1) .{ }^{4}$ Let $\gamma_{X}$ and $\gamma_{Y}$ be smooth, non-intersecting timelike curves in M representing, respectively, the worldlines of $X$ and $Y$. (We will not always bother to distinguish between the curves and their images.) We will follow Rosquist (1980), and define at each point on $\gamma_{X}$ a vector $\Omega^{\mathrm{a}}$ that may be interpreted as the "instantaneous (apparent) angular velocity of $Y$ relative to $X " .5$

Imagine that an observer sitting on particle $\mathrm{X}$ observes particle $\mathrm{Y}$ through a tubular telescope. We can take the orientation of his telescope at a given moment to determine the "(apparent) direction of $\mathrm{Y}$ relative to $\mathrm{X}$ " at that moment; and we can represent the latter as a unit vector, orthogonal to $\gamma_{X}$. In this way, we pass from the 
curves $\gamma_{X}$ and $\gamma_{Y}$ to a (normalized, orthogonal) direction field $v^{\mathrm{a}}$ on $\gamma_{\mathrm{X}}$. Once we have the field $v^{\mathrm{a}}$ in hand, we are almost done. We can then define $\Omega^{\mathrm{a}}$ in terms of $v^{\mathrm{a}}$ in close analogy to the way we previously defined $\vec{\Omega}_{X Y}$ in terms of $\overrightarrow{\mathrm{n}}_{X Y}$. We need only replace the "time derivative" of $\vec{n}_{X Y}$ with the Fermi derivative of $v^{\mathrm{a}}$ along $\gamma_{X}$. Here is the construction in detail. (See figure 1.)

\section{Figure 1}

Let $\xi^{\mathrm{a}}$ be the four-velocity of $\mathrm{X}$, i.e., a future-directed ${ }^{6}$, timelike vector field on $\gamma_{X}$, normalized so that $\xi^{\mathrm{a}} \xi_{\mathrm{a}}=1$. We assume that given any point $\mathrm{p}$ on $\gamma_{X}$, there is (up to reparametrization) a unique future-directed null geodesic that starts at some point (or other) on $\gamma_{Y}$ and ends at $\mathrm{p}$. This amounts to assuming that $\mathrm{X}$ can always see $Y$, and never sees multiple images of $Y{ }^{7}$ Let $\lambda^{\mathrm{a}}$ be the (future directed, null) tangent field to this geodesic (given some choice of parametrization). We arrive at the direction vector $v^{a}$ (of $Y$ relative to $X$ ) at $\mathrm{p}$ by starting with $-\lambda^{\mathrm{a}}$ at that point, then projecting it orthogonal to $\xi^{\mathrm{a}}$, and finally normalizing the resultant vector:

$$
v^{\mathrm{a}}=\frac{-\lambda^{\mathrm{a}}+\left(\lambda^{n_{\xi_{n}}}\right) \xi^{\mathrm{a}}}{\left(\lambda^{\mathrm{m}_{\xi_{m}}}\right)} .
$$

(Equivalently, $v^{\mathrm{a}}$ is the unique vector at $\mathrm{p}$ in the two-plane spanned by $\xi^{\mathrm{a}}$ and $\lambda^{\mathrm{a}}$ such that $v^{\mathrm{a}} \xi_{\mathrm{a}}=0, v^{\mathrm{a}} v_{\mathrm{a}}=-1$, and $v^{\mathrm{a}} \lambda_{\mathrm{a}}>0$.) The Fermi derivative of $v^{\mathrm{a}}$ in the direction $\xi^{\mathrm{a}}$,

$$
\left(\mathrm{g}_{\mathrm{m}}^{\mathrm{a}}-\xi^{\mathrm{a}} \xi_{\mathrm{m}}\right) \xi^{\mathrm{n}} \nabla_{\mathrm{n}} v^{\mathrm{m}}
$$

is just the component of the directional derivative $\xi^{\mathrm{n}} \nabla_{\mathrm{n}} v^{\mathrm{a}}$ orthogonal to $\xi^{\mathrm{a}}$, i.e., the 
spatial component of the derivative as determined relative to $X$. We arrive at the angular velocity $\Omega^{a}$ of $Y$ with respect to $X$ at each point on $\gamma_{X}$, in effect, by taking the cross product there of $v^{\mathrm{a}}$ with $\left(\mathrm{g}^{\mathrm{a}}{ }_{\mathrm{m}}-\xi^{\mathrm{a}} \xi_{\mathrm{m}}\right) \xi^{\mathrm{n}} \nabla_{\mathrm{n}} v^{\mathrm{m}}$ in the three-plane orthogonal to $\xi^{\mathrm{a}}:$

$$
\Omega^{\mathrm{a}}=-\varepsilon^{\mathrm{abcd}} \xi_{\mathrm{b}} v_{\mathrm{c}}\left(\left(\mathrm{g}_{\mathrm{dm}}-\xi_{\mathrm{d}} \xi_{\mathrm{m}}\right) \xi^{\mathrm{n}} \nabla_{\mathrm{n}} \mathrm{v}^{\mathrm{m}}\right)
$$

In analogy to the conditions formulated in section I, we say

(i') $Y$ is not rotating relative to $X$ if

$$
\left.\Omega^{\mathrm{a}}=\mathbf{0} \text { (or, equivalently }{ }^{8},\left(\mathrm{~g}^{\mathrm{a}}{ }_{\mathrm{m}}-\xi^{\mathrm{a}} \xi_{\mathrm{m}}\right) \xi^{\mathrm{n}} \nabla_{\mathrm{n}} v^{\mathrm{m}}=\mathbf{0}\right) \text { at all points on } \gamma_{X^{\prime}}
$$

(ii') $Y$ is rotating relative to $X$ with constant angular velocity if

$$
\left(\mathrm{g}_{\mathrm{m}}^{\mathrm{a}}-\xi^{\mathrm{a}} \xi_{\mathrm{m}}\right) \xi^{\mathrm{n}} \nabla_{\mathrm{n}} \Omega^{\mathrm{m}}=\mathbf{0} \text { at all points on } \gamma_{X} \text {. }
$$

These conditions have a natural physical interpretation. Consider again our observer sitting on particle $X$ and observing $Y$ through his tubular telescope. Condition ( $\left.\mathrm{i}^{\prime}\right)$ holds iff the orientation of his telescope is constant as determined relative to the "compass of inertia". So, for example, we might position three gyroscopes at $\mathrm{X}$ so that their axes are mutually orthogonal. ${ }^{9}$ The orientation of the telescope tube at any moment can then be fully specified by the angles formed between each of the three axes and the tube. Condition (i') captures the requirement that the three angles remain constant. Condition (ii') captures the requirement that the three gyroscopes can be positioned so that the telescope tube is at all times orthogonal to one of the three, and its angles relative to the other two assume the characteristic, sinusoidal pattern of uniform circular motion (with respect to elapsed proper time).

We now consider the special case where $\gamma_{X}$ and $\gamma_{Y}$ are integral curves of a background future-directed, timelike Killing field $\tau^{\mathrm{a}}$. In this case, there is a strong 
sense in which the particles $X$ and $Y$ remain a constant distance apart. ${ }^{10}$ To match our notation above, we express $\tau^{\mathrm{a}}$ in the form $\tau^{\mathrm{a}}=\tau \xi^{\mathrm{a}}$, with $\xi^{\mathrm{a}} \xi_{\mathrm{a}}=1$ and $\tau=$ $\left(\tau^{\mathrm{n}} \tau_{\mathrm{n}}\right)^{1 / 2}$. Associated with $\xi^{\mathrm{a}}$ is a vorticity (or twist) vector field

$$
\omega^{\mathrm{a}}=\frac{1}{2} \varepsilon^{\mathrm{abcd}} \xi_{\mathrm{b}} \nabla_{\mathrm{c}} \xi_{\mathrm{d}}
$$

We want to derive an expression for $\Omega^{\mathrm{a}}$ in terms of $\omega^{\mathrm{a}}$. To do so, we direct attention to the one-parameter group of local isometries $\left\{\Gamma_{\mathrm{s}}\right\}$ associated with $\tau^{\mathrm{a}}$, i.e., the "flow maps" of which $\tau^{\mathrm{a}}$ is the "infinitesimal generator". Given any one null geodesic segment running from $\gamma_{Y}$ to $\gamma_{X}$, it's image under each map $\Gamma_{S}$ is another null geodesic segment running from $\gamma_{Y}$ to $\gamma_{X}$. (This follows immediately. Since $\gamma_{Y}$ and $\gamma_{X}$ are integral curves of $\tau^{\mathrm{a}}$, each is mapped onto itself by $\Gamma_{\mathrm{S}}$. Since $\Gamma_{\mathrm{s}}$ is an isometry, it preserves all structures that can be characterized in terms of the metric $\mathrm{g}_{\mathrm{ab}}$, and that includes the class of null geodesics.) The collection of maps $\left\{\Gamma_{\mathrm{s}}\right\}$ in its entirety, acting on the null geodesic segment, sweeps out a two-dimensional submanifold S, bounded by $\gamma_{Y}$ and $\gamma_{X}$, through every point of which there passes a (unique) integral curve of $\tau^{a}$ and a (unique) null geodesic segment running from $\gamma_{Y}$ and $\gamma_{X}$. (See figure 2.)

\section{Figure 2}

Thus, we have on S two fields tangent to S: the timelike Killing field $\tau^{\mathrm{a}}$, and a future-directed null geodesic field $\lambda^{\mathrm{a}}\left(\lambda^{\mathrm{n}} \nabla_{\mathrm{n}} \lambda^{\mathrm{a}}=0\right.$ and $\left.\lambda^{\mathrm{n}} \lambda_{\mathrm{n}}=0\right)$ that is preserved by each map $\Gamma_{\mathrm{s}^{\prime}}$ or, equivalently, that is Lie derived by the Killing field $\tau^{\mathrm{a}}$, i.e.,

$$
\tau^{\mathrm{n}} \nabla_{\mathrm{n}} \lambda^{\mathrm{a}}-\lambda^{\mathrm{n}} \nabla_{\mathrm{n}} \tau^{\mathrm{a}}=\mathbf{0}
$$


With this equation in hand, it is a matter of routine computation to derive an expression for $\Omega^{\mathrm{a}}$ in terms of $\omega^{\mathrm{a}}$.

Proposition $^{11}$ Let $S, \tau^{\mathrm{a}}$, and $\lambda^{\mathrm{a}}$ be as in the preceding paragraph (and let $\mathrm{v}^{\mathrm{a}}, \Omega^{\mathrm{a}}$, and $\omega^{\mathrm{a}}$ be the corresponding fields on $\mathrm{S}$, as defined earlier in this section). Then, at all points on $S$,

$$
\Omega^{\mathrm{a}}=\omega^{\mathrm{a}}+\left(v^{\mathrm{n}} \omega_{\mathrm{n}}\right) v^{\mathrm{a}}
$$

(We have formulated the proposition in terms of the relative velocity of $Y$ with respect to $\mathrm{X}$. But, of course, a corresponding statement holds if the roles of $\mathrm{X}$ and $\mathrm{Y}$ are reversed. One just has to remember that the reversal brings with it a different two-dimensional submanifold $S$ and a different null field $\lambda^{\mathrm{a}}$.)

Proof Since $\lambda^{\mathrm{a}}, \mathrm{g}_{\mathrm{ab}}$ (and $\tau^{\mathrm{a}}$ ) are Lie derived by the Killing field $\tau^{\mathrm{a}}$, so are all fields definable in terms of them. In particular, $v^{b}$ is Lie derived by $\tau^{\mathrm{a}}$. Thus,

$$
\begin{aligned}
\mathbf{0} & =\tau^{\mathrm{n}} \nabla_{n} v^{\mathrm{m}}-v^{\mathrm{n}} \nabla_{n} \tau^{\mathrm{m}}=\left(\tau \xi^{\mathrm{n}}\right) \nabla_{n} v^{\mathrm{m}}-v^{\mathrm{n}} \nabla_{n}\left(\tau \xi^{\mathrm{m}}\right) \\
& =\left(\tau \xi^{\mathrm{n}}\right) \nabla_{n} v^{\mathrm{m}}-\tau v^{\mathrm{n}} \nabla_{n} \xi^{\mathrm{m}}-\xi^{\mathrm{m}} v^{\mathrm{n}} \nabla_{n} \tau .
\end{aligned}
$$

So

$$
\xi^{\mathrm{n}} \nabla_{\mathrm{n}} v^{\mathrm{m}}=v^{\mathrm{n}} \nabla_{\mathrm{n}} \xi^{\mathrm{m}}+\left(\tau^{-1}\right) \xi^{\mathrm{m}} v^{\mathrm{n}} \nabla_{\mathrm{n}} \tau
$$

and, hence,

$$
\left(g_{d m}-\xi_{d} \xi_{m}\right) \xi^{n} \nabla_{n} v^{m}=\left(g_{d m}-\xi_{d} \xi_{m}\right) v^{n} \nabla_{n} \xi^{m}
$$

since $\left(g_{d m}-\xi_{d} \xi_{m}\right) \xi^{m}=0$. Therefore,

$$
\begin{aligned}
\Omega^{\mathrm{a}} & =-\varepsilon^{\mathrm{abcd}} \xi_{\mathrm{b}} v_{c}\left(\left(g_{d m}-\xi_{d} \xi_{m}\right) \xi^{\mathrm{n}} \nabla_{n} v^{\mathrm{m}}\right)=-\varepsilon^{\mathrm{abcd}} \xi_{\mathrm{b}} v_{c}\left(\left(g_{d m}-\xi_{d} \xi_{m}\right) v^{\mathrm{n}} \nabla_{n} \xi^{\mathrm{m}}\right) \\
& =-\varepsilon^{\mathrm{abcd}} \xi_{\mathrm{b}} v_{c}\left(v^{\mathrm{n}} \nabla_{n} \xi_{d}\right) .
\end{aligned}
$$


The final equality follows from the fact that $\varepsilon^{\mathrm{abcd}} \xi_{\mathrm{b}} \xi_{\mathrm{d}}=\mathbf{0}$ (since $\varepsilon^{\mathrm{abcd}}$ is antisymmetric in the indices ' $b$ ' and 'd'). To proceed further, we use the following expression for $\nabla_{n} \xi_{d}$ that holds for any unit timelike field $\xi^{\mathrm{a}}$ proportional to a Killing field $^{12}$ :

$$
\nabla_{n} \xi_{d}=\varepsilon_{n d p r} \xi^{p} \omega^{r}+\xi_{n} \xi^{m} \nabla_{m} \xi_{d}
$$

Direct substitution yields:

$$
\begin{aligned}
\Omega^{\mathrm{a}} & =-\varepsilon^{\mathrm{abcd}} \xi_{\mathrm{b}} v_{\mathrm{c}} v^{\mathrm{n}}\left(\varepsilon_{\mathrm{ndpr}} \xi^{\mathrm{p}} \omega^{\mathrm{r}}+\xi_{\mathrm{n}} \xi^{\mathrm{m}} \nabla_{\mathrm{m}} \xi_{\mathrm{d}}\right)=-\varepsilon^{\mathrm{abcd}} \xi_{\mathrm{b}} v_{\mathrm{c}} v^{\mathrm{n}} \varepsilon_{n d p r} \xi^{\mathrm{p}} \omega^{\mathrm{r}} \\
& =-\varepsilon^{\mathrm{dabc}} \varepsilon_{d n p r} \xi_{\mathrm{b}} v_{\mathrm{c}} v^{\mathrm{n}} \xi^{\mathrm{p}} \omega^{\mathrm{r}}=6 \delta^{\mathrm{a}}{ }_{[\mathrm{n}} \delta^{\mathrm{b}}{ }_{\mathrm{p}} \delta_{\mathrm{r}]}^{\mathrm{c}} \xi_{\mathrm{b}} v_{\mathrm{c}} v^{\mathrm{n}} \xi^{\mathrm{p}} \omega^{\mathrm{r}} \\
& =6 \xi_{\mathrm{b}} v_{\mathrm{c}} v^{[\mathrm{a}} \xi^{\mathrm{b}} \omega^{\mathrm{c}]}=\xi_{\mathrm{b}} v_{\mathrm{c}}\left(v^{\mathrm{a}} \xi^{\mathrm{b}} \omega^{\mathrm{c}} \ldots-v^{\mathrm{c}} \xi^{\mathrm{b}} \omega^{\mathrm{a}}+\ldots\right) \\
& =\left(v_{\mathrm{c}} \omega^{\mathrm{c}}\right) v^{\mathrm{a}}+\omega^{\mathrm{a}} .
\end{aligned}
$$

(The second equality follows from the fact that $v^{\mathrm{n}} \xi_{\mathrm{n}}=0$; the fourth from the fact that $\varepsilon^{\mathrm{dabc}} \varepsilon_{\mathrm{dnpr}}=-6 \delta_{[\mathrm{n}}^{\mathrm{a}} \delta_{\mathrm{p}}^{\mathrm{b}} \delta_{\mathrm{r}]}^{\mathrm{c}}$. For the latter, see Wald (1984), p. 432.) $/ /$

We claimed above that relativity theory allows for the possibility that there be two point particles $\mathrm{X}$ and $\mathrm{Y}$, a constant distance apart, such that $\mathrm{Y}$ is non-rotating relative to $\mathrm{X}$, but $\mathrm{X}$ is rotating relative to $\mathrm{Y}$ with constant (non-zero) angular velocity. Our strategy for producing an example in section III is this. We exhibit a spacetime with a future-directed, timelike Killing field $\tau^{\mathrm{a}}=\tau \xi^{\mathrm{a}}$, and two integral curves of the field, $\gamma_{X}$ and $\gamma_{Y}$, such that the following conditions hold.

(a) $\omega^{\mathrm{a}}=\mathbf{0}$ on $\gamma_{\mathrm{X}}$.

(b) $\omega^{\mathrm{a}} \neq 0$ on $\gamma_{Y}$, but $\left(\mathrm{g}_{\mathrm{m}}^{\mathrm{a}}-\xi^{\mathrm{a}} \xi_{\mathrm{m}}\right) \xi^{\mathrm{n}} \nabla_{\mathrm{n}} \omega^{\mathrm{m}}=\mathbf{0}$ on $\gamma_{Y}$.

(c) Whether working from $\gamma_{Y}$ to $\gamma_{X}$, or from $\gamma_{X}$ to $\gamma_{Y}$, the associated future- 
directed null geodesic field $\lambda^{\mathrm{a}}$ that is Lie derived by $\tau^{\mathrm{a}}$ (as in the construction above) is everywhere orthogonal to $\omega^{\mathrm{a}}$.

This will suffice. Consider the condition in (c). If the connecting null field $\lambda^{a}$ is orthogonal to $\omega^{\mathrm{a}}$, then the direction field $v^{\mathrm{a}}$ induced by $\lambda^{\mathrm{a}}$ is also orthogonal to $\omega^{\mathrm{a}}$ :

$$
v^{\mathrm{a}} \omega_{\mathrm{a}}=\left(\frac{-\lambda^{\mathrm{a}}+\left(\lambda^{n_{\xi_{n}}}\right) \xi^{\mathrm{a}}}{\left(\lambda^{m_{\xi_{m}}}\right)}\right) \omega_{\mathrm{a}}=\xi^{\mathrm{a}} \omega_{\mathrm{a}}=0 .
$$

So, by the proposition, $\Omega^{\mathrm{a}}{ }_{(\mathrm{Y} \text { wrt X) }}=\omega^{\mathrm{a}}$ on $\gamma_{X^{\prime}}$ and $\Omega^{\mathrm{a}}{ }_{\left(\mathrm{X}_{\text {wrt }} \mathrm{Y}\right)}=\omega^{\mathrm{a}}$ on $\gamma_{\mathrm{Y}}$.

So by (a) and (b),

$$
\begin{aligned}
& \Omega_{(Y \text { wrt } X)}^{\mathrm{a}}=\mathbf{0} \text { on } \gamma_{X}, \text { while } \\
& \Omega_{\left(X_{\text {wrt }}\right)}^{\mathrm{a})} \neq \mathbf{0} \text { on } \gamma_{Y^{\prime}} \text { but }\left(\mathrm{g}^{\mathrm{a}}{ }_{\mathrm{m}}-\xi^{\mathrm{a}} \xi_{\mathrm{m}}\right) \xi^{\mathrm{n}} \nabla_{\mathrm{n}} \Omega^{\mathrm{m}}{ }_{\left(\mathrm{X}_{\text {wrt } \mathrm{Y}}\right)}=\mathbf{0} \text { on } \gamma_{Y^{\prime}}
\end{aligned}
$$

as desired.

III

The example we present in this section is a bit artificial. But it does have the virtue of simplicity. It will be relatively easy to identify the necessary elements of structure -- the timelike Killing field $\tau^{\mathrm{a}}$, and the integral curves $\gamma_{X}$ and $\gamma_{Y^{--}}$and verify that they satisfy conditions (a)-(c). Given how very stringent the conditions are, it is of some interest, perhaps, to have any simple example at all.

In constructing the example, we start with Gödel spacetime $\left(\mathrm{M}, \mathrm{g}_{\mathrm{ab}}\right)$ in its entirety and then, at a certain point, shift attention to a restricted model of form $\left(\mathrm{O}, \mathrm{g}_{\mathrm{ab}} \mid \mathrm{O}\right)$, where $\mathrm{O}$ is an open subset of $\mathrm{M}$. The restricted spacetime is, in some respects, much better behaved than the original. In particular, it does not admit closed timelike curves. Indeed, it satisfies the stable causality condition. (But, unlike the original, of course, it is extendible.) 
In what follows, we take Gödel spacetime ${ }^{13}$ to be the pair $\left(M, g_{a b}\right)$, where $M$ is the manifold ${ }^{4}$ and $g_{a b}$ is characterized by the condition that given any point $\mathrm{p}$ in $\mathrm{M}$, there is a global (adapted) cylindrical coordinate system $t, r, \varphi$, y on $M$ such that $t(p)$ $=\mathrm{r}(\mathrm{p})=\mathrm{y}(\mathrm{p})=0$ and

$$
\begin{aligned}
g_{a b}=4 \mu^{2}\left[(d t)_{a}(d t)_{b}-(d r)_{a}(d r)_{b}-(d y)_{a}(d y)_{b}\right. \\
\left.+\left(s h^{4} r-s h^{2} r\right)(d \varphi)_{a}(d \varphi)_{b}+\sqrt{2} s h^{2} r\left((d t)_{a}(d \varphi)_{b}+(d \varphi)_{a}(d t)_{b}\right)\right] .
\end{aligned}
$$

(We use 'sh r' and 'ch r' to stand for 'sinh r' and 'cosh r'.) Here $-\infty<t<\infty$, $0 \leq \mathrm{r}<\infty,-\infty<\mathrm{y}<\infty$, and $0 \leq \varphi<\infty$ with $\varphi=0$ identified with $\varphi=2 \pi ; \mu$ is an arbitrary positive constant. (We will assume a point $\mathrm{p}$ has been chosen, once and for all, and work with the corresponding coordinate system.) The metric $g_{a b}$ is a solution to Einstein's equation

$$
R_{a b}-(1 / 2) g_{a b} R=8 \pi G T_{a b}
$$

for a perfect fluid source

$$
\mathrm{T}_{\mathrm{ab}}=\rho \eta_{\mathrm{a}} \eta_{\mathrm{b}}-\mathrm{p}\left(\mathrm{g}_{\mathrm{ab}}-\eta_{\mathrm{a}} \eta_{\mathrm{b}}\right)
$$

with four-velocity $\eta^{\mathrm{a}}=(2 \mu)^{-1}(\partial / \partial \mathrm{t})^{\mathrm{a}}$, mass density $\rho=\left(16 \pi G \mu^{2}\right)^{-1}$, and isotropic pressure $p=\left(16 \pi G \mu^{2}\right)^{-1}$.

The field $(\partial / \partial \mathrm{t})^{\mathrm{a}}$ is everywhere timelike, and defines a temporal orientation on $\left(\mathrm{M}, \mathrm{g}_{\mathrm{ab}}\right)$. The integral curves of $(\partial / \partial \mathrm{t})^{\mathrm{a}}$ will be called "matter lines" (since the fourvelocity $\eta^{\mathrm{a}}$ of the fluid source is everywhere proportional to $\left.(\partial / \partial \mathrm{t})^{\mathrm{a}}\right)$.

In the appendix, we give an explicit expression for a volume element $\varepsilon^{\text {abcd }}$ on $\left(\mathrm{M}, \mathrm{g}_{\mathrm{ab}}\right)$ in terms of coordinates $\mathrm{t}, \mathrm{r}, \varphi, \mathrm{y}$. It defines an orientation on $\left(\mathrm{M}, \mathrm{g}_{\mathrm{ab}}\right)$.

In Gödel spacetime, $(\partial / \partial \mathrm{t})^{\mathrm{a}},(\partial / \partial \mathrm{y})^{\mathrm{a}}$, and $(\partial / \partial \varphi)^{\mathrm{a}}$ are all Killing fields and so, 
therefore, are all linear combinations of these fields. We will be interested, specifically, in the field

$$
\tau^{\mathrm{a}}=(\partial / \partial \mathrm{t})^{\mathrm{a}}+\sqrt{2}(\partial / \partial \varphi)^{\mathrm{a}}
$$

Since

$$
\tau^{\mathrm{a}} \tau_{\mathrm{a}}=4 \mu^{2}\left[1+2\left(\operatorname{sh}^{4} \mathrm{r}-\mathrm{sh}^{2} \mathrm{r}\right)+4 \mathrm{sh}^{2} \mathrm{r}\right]=4 \mu^{2}\left[1+2\left(\mathrm{sh}^{2} \mathrm{r}\right)\left(\mathrm{ch}^{2} \mathrm{r}\right)\right]
$$

it follows immediately that

(1) $\tau^{\mathrm{a}}$ is everywhere timelike.

It is also clear that

(2) the coordinate functions $\mathrm{r}$ and $\mathrm{y}$ are constant on all integral curves of $\tau^{\mathrm{a}}{ }^{14}$

If the constant value of $\mathrm{r}$ is 0 , the integral curve is a matter line (since $(\partial / \partial \varphi)^{\mathrm{a}}=\mathbf{0}$ where $r=0$ ), characterized by its y value. We call it an "axis curve". If the constant value of $r$ is strictly positive, we can picture it as a helix that wraps around an axis curve (the one with the same y value). ${ }^{15}$

If, as above, we express $\tau^{\mathrm{a}}$ in the form $\tau^{\mathrm{a}}=\tau \xi^{\mathrm{a}}$, with $\xi^{\mathrm{a}} \xi_{\mathrm{a}}=1$, the vorticity field associated with $\xi^{\mathrm{a}}$ comes out to be:

(3) $\omega^{\mathrm{a}}=\frac{2 \sqrt{2}\left(\mathrm{sh}^{2} \mathrm{r}\right)\left(\mathrm{ch}^{2} \mathrm{r}\right)}{\left(4 \mu^{2}\right)\left[1+2\left(\operatorname{sh}^{2} \mathrm{r}\right)\left(\mathrm{ch}^{2} \mathrm{r}\right)\right]}(\partial / \partial \mathrm{y})^{\mathrm{a}}$

(The computation requires just a bit of work. We present it in the appendix.) It follows immediately that

(4) $\omega^{\mathrm{a}}=\mathbf{0} \Leftrightarrow \mathrm{r}=0 .{ }^{16}$

It also follows that 
(5) $\left(\mathrm{g}_{\mathrm{m}}^{\mathrm{a}}-\xi^{\mathrm{a}} \xi_{\mathrm{m}}\right) \xi^{\mathrm{n}} \nabla_{\mathrm{n}} \omega^{\mathrm{m}}=\mathbf{0}$ everywhere.

In fact, the stronger condition $\xi^{\mathrm{n}} \nabla_{n} \omega^{\mathrm{a}}=0$ holds everywhere. This follows because $\xi^{\mathrm{n}} \nabla_{\mathrm{n}} \mathrm{r}=0$, by (2) above, and $(\partial / \partial \mathrm{y})^{\mathrm{a}}$ is covariantly constant, i.e., $\nabla_{\mathrm{n}}(\partial / \partial \mathrm{y})^{\mathrm{a}} \cdot{ }^{17}$ So

$$
\xi^{\mathrm{n}} \nabla_{\mathrm{n}} \omega^{\mathrm{a}}=\frac{2 \sqrt{2}\left(\operatorname{sh}^{2} \mathrm{r}\right)\left(\operatorname{ch}^{2} \mathrm{r}\right)}{\left(4 \mu^{2}\right)\left[1+2\left(\operatorname{sh}^{2} \mathrm{r}\right)\left(\mathrm{ch}^{2} \mathrm{r}\right)\right]} \xi^{\mathrm{n}} \nabla_{\mathrm{n}}(\partial / \partial \mathrm{y})^{\mathrm{a}}=\mathbf{0} .
$$

We are now well on our way. If we take $\gamma_{X}$ to be any integral curve of $\tau^{\mathrm{a}}$ with $r=0$, and $\gamma_{Y}$ to be any one with $r \neq 0$, conditions (a) and (b) listed at the end of section II will be automatically satisfied. So it only remains to consider condition (c).

To satisfy the orthogonality constraint in (c), we need to further restrict the choice of $\gamma_{X}$ and $\gamma_{Y}$ so that the y coordinate function has the same (constant) value on both curves. Let $\mathrm{r}_{\mathrm{o}}$ be any positive real number and let $\mathrm{y}_{\mathrm{o}}$ be any real whatsoever. Let $\gamma_{X}$ be an integral curve of $\tau^{\mathrm{a}}$ with constant values $r=0, y=y_{O^{\prime}}$ and let $\gamma_{Y}$ be one with constant values $\mathrm{r}=\mathrm{r}_{\mathrm{o}^{\prime}} \mathrm{y}=\mathrm{y}_{\mathrm{o}}$. The following conditional claim about null geodesics follows easily.

(6) If there exists a null geodesic that intersects both $\gamma_{Y}$ and $\gamma_{X}$, and if $\lambda^{a}$ is the tangent field to the curve, then $\lambda^{a} \omega_{a}=0$ at all points on the curve.

For assume there is such a curve $\sigma$ with tangent field $\lambda^{\mathrm{a}}$. Since $\lambda^{\mathrm{a}}$ is a geodesic field, we have $\lambda^{\mathrm{n}} \nabla_{\mathrm{n}}\left(\lambda^{\mathrm{a}} \kappa_{\mathrm{a}}\right)=0$ for all Killing fields $\kappa^{\mathrm{a}}$. ${ }^{18}$ In particular, taking $\kappa^{\mathrm{a}}$ to be $(\partial / \partial y)^{\mathrm{a}}, \lambda^{\mathrm{a}}(\partial / \partial \mathrm{y})_{\mathrm{a}}$ is constant on $\sigma$. But $\lambda^{\mathrm{a}}(\partial / \partial \mathrm{y})_{\mathrm{a}}=-4 \mu^{2}\left(\lambda^{\mathrm{a}} \nabla_{\mathrm{a}} \mathrm{y}\right)$. So $\lambda^{\mathrm{a}} \nabla_{\mathrm{a}} \mathrm{y}$ is constant on $\sigma$. If the constant value of this function were not 0 , the value of the coordinate y would have to increase or decrease along $\sigma$-- contradicting the fact that the initial and final points share the value $y_{0}$. So it must be the case that $\lambda^{a}(\partial / \partial y)_{a}$ is 0 at all points on $\sigma$. But, by (3), $\omega^{\mathrm{a}}$ is everywhere proportional to $(\partial / \partial y)^{\mathrm{a}}$. So $\lambda^{\mathrm{a}} \omega_{\mathrm{a}}=0$ at all points on $\sigma$.

Now it only remains for us to consider the existence and uniqueness of null 
geodesics running between $\gamma_{Y}$ and $\gamma_{X}$. But here, for the first time, things get sticky. We want to be able to assert that an observer on one of the particles will see the other at all times, but not see it in more than one position on the celestial sphere. It is a curious fact about null geodesics in Gödel spacetime that this will simply not be the case, in general. It turns out that if $s h r_{o}>1$ (i.e., if $r_{o}>\ln (1+\sqrt{2})$ ), the observer will not see the other particle at all. And if $s h r_{o} \leq 1$, he will, in general, see multiple images of the other. Roughly speaking, this results from the fact that photons act like boomerangs in Gödel spacetime. Any future or past directed null geodesic that starts at a point on $\gamma_{X}$ moves outward (with monotonically increasing $r$ value) until it reaches the critical radius $r_{c}=\ln (1+\sqrt{2})$, and then moves inward (with monotonically decreasing $r$ value) until it hits $\gamma_{X}$ again; and then the process starts all over. ${ }^{19}$ So, it can happen, for example, that two past-directed null geodesics start out in different directions from a point on $\gamma_{X}$, and both intersect $\gamma_{Y}$, though at different points. One hits $\gamma_{Y}$ on the way out. The other hits it on the return trip in.

To avoid this complication, we now impose the requirement that $\mathrm{r}_{\mathrm{o}}<\mathrm{r}_{\mathrm{C}^{\prime}}$ restrict attention to the open subset

$$
O=\left\{q \in M: r(q)<r_{c}\right\}
$$

and consider $\left(\mathrm{O}, \mathrm{g}_{\mathrm{ab}} \mathrm{O}_{\mathrm{O}}\right)$ as a spacetime model in its own right (with the temporal orientation and orientation inherited from the original). ${ }^{20}$ Then we can make the desired existence and uniqueness claim concerning null geodesics.

(7) Given any point $q_{X}$ on $\gamma_{X}$, there is a unique point $q_{Y}$ on $\gamma_{Y}$ such that there exists a future directed null geodesic running from $\mathrm{q}_{Y}$ to $\mathrm{q}_{X}$; and symmetrically, with the roles of $\mathrm{X}$ and $\mathrm{Y}$ reversed.

That this is true follows alone from the qualitative description of past and future 
directed null geodesics just given (the boomerang effect). We sketch the proof in the appendix.

This puts all the needed pieces of the example together. We now revert to the discussion at the end of section II.

\section{Appendix: Needed Facts About Gödel Spacetime}

(A) Derivation of formula (3) in section III

Let $\mathrm{k}$ be any real number and let $\tau^{\mathrm{a}}$ be the Killing field $(\partial / \partial \mathrm{t})^{\mathrm{a}}+\mathrm{k}(\partial / \partial \varphi)^{\mathrm{a}}$. If we restrict attention to the (open) region where it is timelike, we can express $\tau^{\mathrm{a}}$ in the form $\tau^{\mathrm{a}}=\tau \xi^{\mathrm{a}}$, with $\xi^{\mathrm{a}} \xi_{\mathrm{a}}=1$. We claim that the vorticity associated with $\xi^{\mathrm{a}}$ (in this region) is given by

$$
\omega^{\mathrm{a}}=\frac{\sqrt{2}+\mathrm{k}\left(2 \mathrm{sh}^{2} \mathrm{r}-1\right)+\mathrm{k}^{2} \sqrt{2} \mathrm{sh}^{4} \mathrm{r}}{\left(4 \mu^{2}\right)\left[1+\mathrm{k} 2 \sqrt{2} \mathrm{sh}^{2} \mathrm{r}+\mathrm{k}^{2}\left(\mathrm{sh}^{4} \mathrm{r}-\mathrm{sh}^{2} \mathrm{r}\right)\right]}(\partial / \partial \mathrm{y})^{\mathrm{a}} .
$$

If $k=\sqrt{2}$, this reduces to (3).

In the derivation, we use the following basic relations:

$$
\begin{gathered}
(\partial / \partial \mathrm{t})_{\mathrm{a}}=4 \mu^{2}\left((\mathrm{dt})_{\mathrm{a}}+\sqrt{2} \operatorname{sh}^{2} \mathrm{r}(\mathrm{d} \varphi)_{\mathrm{a}}\right) \\
(\partial / \partial \varphi)_{\mathrm{a}}=4 \mu^{2}\left(\sqrt{2} \operatorname{sh}^{2} \mathrm{r}(\mathrm{dt})_{\mathrm{a}}+\left(\operatorname{sh}^{4} \mathrm{r}-\mathrm{sh}^{2} \mathrm{r}\right)(\mathrm{d} \varphi)_{\mathrm{a}}\right) \\
\tau_{\mathrm{a}}=4 \mu^{2}\left(\left(1+\mathrm{k} \sqrt{2} \operatorname{sh}^{2} \mathrm{r}\right)(\mathrm{dt})_{\mathrm{a}}+\left(\sqrt{2} \operatorname{sh}^{2} \mathrm{r}+\mathrm{k}\left(\operatorname{sh}^{4} \mathrm{r}-\operatorname{sh}^{2} \mathrm{r}\right)\right)(\mathrm{d} \varphi)_{\mathrm{a}}\right) \\
\tau^{2}=\tau^{\mathrm{a}} \tau_{\mathrm{a}}=4 \mu^{2}\left(1+\mathrm{k} 2 \sqrt{2} \operatorname{sh}^{2} \mathrm{r}+\mathrm{k}^{2}\left(\operatorname{sh}^{4} \mathrm{r}-\mathrm{sh}^{2} \mathrm{r}\right)\right),
\end{gathered}
$$

and we work with the volume element defined ${ }^{21}$ by

$$
\varepsilon^{\mathrm{abcd}}=\mathrm{f}(\partial / \partial \mathrm{t})^{[\mathrm{a}}(\partial / \partial \mathrm{r})^{\mathrm{b}}(\partial / \partial \varphi)^{\mathrm{c}}(\partial / \partial \mathrm{y})^{\mathrm{d}]} \text { where } \mathrm{f}=\frac{-4 !}{\left(16 \mu^{4}\right)(\operatorname{sh~} \mathrm{r})(\mathrm{ch} \mathrm{r})} .
$$


Since $\varepsilon^{\mathrm{abcd}} \tau_{\mathrm{b}} \tau_{\mathrm{d}}=0$, we have

$$
\omega^{\mathrm{a}}=\frac{1}{2} \varepsilon^{\mathrm{abcd}} \xi_{\mathrm{b}} \nabla_{\mathrm{c}} \xi_{\mathrm{d}}=\frac{1}{2} \varepsilon^{\mathrm{abcd}} \frac{\tau_{\mathrm{b}}}{\tau} \nabla_{\mathrm{c}} \frac{\tau_{\mathrm{d}}}{\tau}=\frac{1}{2 \tau^{2}} \varepsilon^{\mathrm{abcd}} \tau_{[\mathrm{b}} \nabla_{\mathrm{c}} \tau_{\mathrm{d}]} .
$$

So we start by deriving an expression for $\tau_{[b} \nabla_{c} \tau_{d]}$. First, note that since $\nabla_{[c}(d t)_{d]}=0$ $=\nabla_{[\mathrm{c}}(\mathrm{d} \varphi)_{\mathrm{d}]}$

$$
\begin{aligned}
\nabla_{[\mathrm{c}} \tau_{\mathrm{d}]}= & 4 \mu^{2}\left((\mathrm{dt})_{[\mathrm{d}} \nabla_{\mathrm{c}]}\left(1+\mathrm{k} \sqrt{2} \operatorname{sh}^{2} \mathrm{r}\right)+(\mathrm{d} \varphi)_{[\mathrm{d}} \nabla_{\mathrm{c}]}\left(\sqrt{2} \operatorname{sh}^{2} \mathrm{r}+\mathrm{k}\left(\mathrm{sh}^{4} \mathrm{r}-\mathrm{sh}^{2} \mathrm{r}\right)\right)\right) \\
= & 4 \mu^{2}\left(\mathrm{k} 2 \sqrt{2}(\mathrm{sh})(\mathrm{ch} \mathrm{r})(\mathrm{dt})_{[\mathrm{d}}(\mathrm{dr})_{\mathrm{c}]}+\right. \\
& \left.2(\operatorname{sh~r})(\mathrm{ch} \mathrm{r})\left(\sqrt{2}+\mathrm{k}\left(2 \operatorname{sh}^{2} \mathrm{r}-1\right)\right)(\mathrm{d} \varphi)_{[\mathrm{d}}(\mathrm{dr})_{\mathrm{c}]}\right) .
\end{aligned}
$$

Hence,

$$
\tau_{[b} \nabla_{c} \tau_{d]}=\tau_{[b} \nabla_{[c} \tau_{d]]}=\left(16 \mu^{4}\right) K(d t)_{[b}(d r)_{c}(d \varphi)_{d]}
$$

where

$$
\begin{aligned}
& K=-k 2 \sqrt{2}(\operatorname{sh~} r)(\operatorname{ch~} r)\left(\sqrt{2} s^{2} r+k\left(s h^{4} r-s h^{2} r\right)\right) \\
& +\left(1+k \sqrt{2} \operatorname{sh}^{2} r\right) 2(\operatorname{sh} r)(\operatorname{ch~} r)\left(\sqrt{2}+k\left(2 s^{2} r-1\right)\right) \\
& =2(\operatorname{sh~} r)(\operatorname{ch~r})\left[\sqrt{2}+\mathrm{k}\left(2 \operatorname{sh}^{2} \mathrm{r}-1\right)+\mathrm{k}^{2} \sqrt{2} \operatorname{sh}^{4} \mathrm{r}\right] .
\end{aligned}
$$

So,

$$
\begin{aligned}
\omega^{\mathrm{a}} & =\frac{1}{2 \tau^{2}} \varepsilon^{\mathrm{abcd}} \tau_{[\mathrm{b}} \nabla_{\mathrm{c}} \tau_{\mathrm{d}]}=\frac{1}{2 \tau^{2}}\left(16 \mu^{4}\right) \mathrm{K} \varepsilon^{\mathrm{abcd}}(\mathrm{dt})_{[\mathrm{b}}(\mathrm{dr})_{\mathrm{c}}(\mathrm{d} \varphi)_{\mathrm{d}]} \\
& =\frac{1}{2 \tau^{2}}\left(16 \mu^{4}\right) \mathrm{K} \mathrm{f}(4 !)^{-1}\left(-(\partial / \partial \mathrm{y})^{\mathrm{a}}\right) \\
& =\frac{\sqrt{2}+\mathrm{k}\left(2 \operatorname{sh}^{2} \mathrm{r}-1\right)+\mathrm{k}^{2} \sqrt{2} \operatorname{sh}^{4} \mathrm{r}}{\left(4 \mu^{2}\right)\left[1+\mathrm{k} 2 \sqrt{2} \mathrm{sh}^{2} \mathrm{r}+\mathrm{k}^{2}\left(\mathrm{sh}^{4} \mathrm{r}-\mathrm{sh}^{2} \mathrm{r}\right)\right]}(\partial / \partial \mathrm{y})^{\mathrm{a}} . / /
\end{aligned}
$$

(B) Proof sketch of claim (7) in section III

Let $\mathrm{q}_{X}$ be any point on $\gamma_{X}$ and let $\tilde{\lambda}^{\mathrm{a}}$ be any past-directed (non-zero) null vector at $\mathrm{q}_{\mathrm{X}}$ such that $\tilde{\lambda}^{\mathrm{n}} \nabla_{\mathrm{n}} \mathrm{y}=0$. Let $\sigma$ be the (unique) inextendible, past-directed null 
geodesic starting at $q_{X}$ whose tangent at that point is $\tilde{\lambda}^{\mathrm{a}}$. Let its tangent field be $\lambda^{\mathrm{a}}$. The $\mathrm{r}$ coordinate on $\sigma$ starts at 0 and increases (monotonically) through all values less than $\mathrm{r}_{\mathrm{c}}$. So there is exactly one point $\mathrm{q}$ on $\sigma$ whose $\mathrm{r}$ value is $\mathrm{r}_{\mathrm{o}}{ }^{22}$ Let the coordinates of $\mathrm{q}$ be $\left(\mathrm{t}, \mathrm{r}_{\mathrm{o}^{\prime}} \varphi, \mathrm{y}_{\mathrm{o}}\right)$. (We know, from the discussion after (5), that $\lambda^{\mathrm{n}} \nabla_{\mathrm{n}} \mathrm{y}$ is constant on $\sigma$. Since it is 0 at $q_{X}$, it must be 0 at all points. So the value of the $y$ coordinate must be $\mathrm{y}_{\mathrm{o}}$ at all points on $\sigma$.) The point q need not fall on $\gamma_{Y}$.

We have so far considered just one inextendible, past-directed null geodesic starting at $\mathrm{q}_{\mathrm{X}}$ along which $\mathrm{y}$ has the constant value $\mathrm{y}_{\mathrm{O}}$. But the entire class of these is generated by taking the image of $\sigma$ under "rotations" of form

$$
(\mathrm{t}, \mathrm{r}, \varphi, \mathrm{y}) \rightarrow\left(\mathrm{t}, \mathrm{r}, \varphi+\varphi_{\mathrm{O}^{\prime}} \mathrm{y}\right)
$$

i.e., under isometries generated by the Killing field $(\partial / \partial \varphi)^{\mathrm{a}}$. One of these isometric images of $\sigma$ does intersect $\gamma_{Y}$ (since there is some point $q_{Y}$ on $\gamma_{Y}$ and some $\varphi_{\mathrm{O}}$ such that $\mathrm{q}_{Y}$ has coordinates $\left(\mathrm{t}, \mathrm{r}_{\mathrm{O}^{\prime}} \varphi+\varphi_{\mathrm{O}^{\prime}} \mathrm{y}_{\mathrm{O}}\right)$ ). The time reversed, i.e., future-directed, version of this curve qualifies as a null geodesic running from a point $q_{Y}$ on $\gamma_{Y}$ to $q_{X}$. So we have established the existence claim in (7). And uniqueness follows easily as well. Suppose $\sigma_{1}$ and $\sigma_{2}$ are both past-directed null geodesics starting at $q_{X}$ that intersect $\gamma_{Y}$. Then since both arise as images of $\sigma$ under rotations of the sort just described, and since these maps preserve the value of the coordinate $t$, the intersection points share a common value of $\mathrm{t}$. But there can be only one point on $\gamma_{Y}$ having any particular value of t. (This follows because $\gamma_{Y}$ is a future directed timelike curve, and (see note 19) the coordinate function $t$ is strictly increasing on all such curves.)

The argument for the symmetric claim (with the roles of $X$ and $Y$ interchanged) is very much the same. But now, in addition to considering "rotations" (as above), we also consider "timelike translations" of form 


$$
(t, r, \varphi, y) \rightarrow\left(t+t_{o^{\prime}} r, \varphi, y\right)
$$

i.e., isometries generated by the Killing field $(\partial / \partial t)^{a}$. Let $q_{Y}$ be any point on $\gamma_{Y}$. Essentially the same argument as we have just considered shows that given any point on $\gamma_{X}$, there is a unique point on $\gamma_{Y}$ such that there exists a future-directed null geodesic running from the first point to the second. By moving to the image of this curve under, first, a timelike translation and, then, a rotation, we arrive at a future-directed null geodesic $\sigma$ that starts at a point $\mathrm{q}_{X}$ on $\gamma_{X}$ and ends at $\mathrm{q}_{Y}$. This gives us existence. For uniqueness, suppose there were a second point $\mathrm{q}_{X}^{\prime}$ on $\gamma_{X}$ and a null geodesic $\sigma^{\prime}$ running from $\mathrm{q}_{X}^{\prime}{ }_{X}$ to $q_{Y}$. By first sliding $\sigma^{\prime}$ up or down so that $q_{X}^{\prime}$ is mapped to $q_{X}$, and then rotating it, we could generate a future-directed null geodesic that starts at $\mathrm{q}_{X}$, but ends at a point on $\gamma_{Y}$ distinct from $\mathrm{q}_{Y^{--}}$and this we know is impossible. // 


\section{$\underline{\text { Footnotes }}$}

1 It is a pleasure to dedicate this paper to John Stachel, and thank him here for the encouragement and support he has given me over the years. (It is also a pleasure to thank Robert Geroch, Howard Stein, and Robert Wald for helpful comments on an earlier draft.)

2 It should be emphasized that this does not imply that all claims about "orbital rotation" are symmetric within the framework of Newtonian physics. For example, let $\mathrm{X}$ be a particle sitting at the center of mass of the solar system. The earth and the sun both rotate relative to $\mathrm{X}$ (and relative to each other) in our sense; and $\mathrm{X}$ rotates relative to both the earth and the sun in that sense. But there is this asymmetry between the motion of $\mathrm{X}$ on the one hand, and that of the earth and the sun on the other: $\mathrm{X}$ is non-accelerating, while both the earth and the sun have non-zero acceleration vectors that point toward $\mathrm{X}$. This captures one sense in which one might say that the earth and sun are rotating around $X$, but not conversely.

3 The discussion to this point has been cast in terms of textbook Newtonian particle mechanics. It might be asked what, if anything, changes when one passes to the Cartan formulation of Newtonian theory in which gravity is treated as a manifestation of spacetime curvature. (Rather than thinking of point particles as being deflected from their natural straight trajectories by the presence of a gravitational potential, one thinks of them as traversing the geodesics of a non-flat affine connection.) The short answer is that our notion of relative angular velocity carries over in a natural way, and conditions (i) and (ii) remain symmetric. (More problematic is the notion of orbital rotation considered in the preceding note since it makes reference to the "acceleration" of particles in a gravitational field. But it can 
be reformulated (in terms of the presence of background spacetime symmetries) and remains asymmetric).) It would take us too far afield to sort this all out here.

4 Definitions of the technical terms used here and in what follows can be found, for example, in Wald (1984). (Strictly speaking, a few minor transpositions will be necessary since Wald works with the signature $(-1,+1,+1,+1)$ rather than ours.)

5 We might have written ' $\Omega X Y^{\mathrm{a}}$ ', but that notation is potentially misleading. The identification indices ' $X Y$ ' should not be confused with tensor or spinor indices. In what follows, it will usually be clear from context whether we are talking about the angular velocity of $\mathrm{Y}$ relative to $\mathrm{X}$, or of $\mathrm{X}$ relative to $\mathrm{Y}$. But when there is danger of confusion, we will write ' $\Omega^{\mathrm{a}}{ }_{(\mathrm{Y} \text { wrt X) }}$ ' or ' $\Omega^{\mathrm{a}}{ }_{(\mathrm{X} \text { wrt } \mathrm{Y})}$ '.

6 In what follows, we assume that $\left(\mathrm{M}, \mathrm{g}_{\mathrm{ab}}\right)$ is temporally orientable and a particular temporal orientation has been selected. We also assume that it is orientable and a volume element $\varepsilon_{\mathrm{abcd}}$ has been selected. (A smooth field $\varepsilon_{\mathrm{abcd}}$ on M qualifies as a volume element if it is completely anti-symmetric $\left(\varepsilon_{\text {abcd }}=\varepsilon_{\text {[abcd] }}\right)$ and normalized so that $\varepsilon^{\mathrm{abcd}} \varepsilon_{\mathrm{abcd}}=-4$ !) Neither the assumption of temporal orientability nor orientability is really necessary. We can, alternatively, restrict attention to appropriate local neighborhoods of M. But the assumptions are convenient and, in fact, the spacetime we will use for our example in section III (Gödel spacetime) is temporally orientable and orientable.

7 This is a substantive assumption, and will play a role in the presentation of our example in section III.

8 The equivalence here corresponds perfectly to that in (i) in section I, and the proof is essentially the same. $\Omega^{\mathrm{a}}=0$ iff the three vectors $\xi^{\mathrm{a}}, v^{\mathrm{a}}$, and $\left(\left(\mathrm{g}_{\mathrm{m}}^{\mathrm{a}}-\xi^{\mathrm{a}} \xi_{\mathrm{m}}\right) \xi^{\mathrm{n}} \nabla_{\mathrm{n}} v^{\mathrm{m}}\right.$ are linearly dependent. But since $\xi^{\mathrm{a}}$ and $v^{\mathrm{a}}$ are non-zero, and 
both $v^{\mathrm{a}}$ and $\left(\left(\mathrm{g}_{\mathrm{m}}^{\mathrm{a}}-\xi^{\mathrm{a}} \xi_{\mathrm{m}}\right) \xi^{\mathrm{n}} \nabla_{\mathrm{n}} \mathrm{v}^{\mathrm{m}}\right.$ are orthogonal to $\xi^{\mathrm{a}}$, this condition holds iff $\left(\mathrm{g}^{\mathrm{a}}{ }_{\mathrm{m}}-\xi^{\mathrm{a}} \xi_{\mathrm{m}}\right) \xi^{\mathrm{n}} \nabla_{\mathrm{n}} \mathrm{v}^{\mathrm{m}}$ is proportional to $v^{\mathrm{a}}$. But $\left(\mathrm{g}^{\mathrm{a}} \mathrm{m}^{-} \xi^{\mathrm{a}} \xi_{\mathrm{m}}\right) \xi^{\mathrm{n}} \nabla_{\mathrm{n}} \mathrm{v}^{\mathrm{m}}$ is orthogonal to $v^{\mathrm{a}}$ (since $v_{\mathrm{a}} \xi^{\mathrm{a}}=0$ and $\left.v_{\mathrm{m}} \xi^{\mathrm{n}} \nabla_{\mathrm{n}} \mathrm{v}^{\mathrm{m}}=(1 / 2) \xi^{\mathrm{n}} \nabla_{\mathrm{n}}\left(v^{\mathrm{m}} v_{\mathrm{m}}\right)=(1 / 2) \xi^{\mathrm{n}} \nabla_{\mathrm{n}}(-1)=0\right)$. So $\Omega^{\mathrm{a}}=\mathbf{0}$ iff $\left(\mathrm{g}_{\mathrm{m}}^{\mathrm{a}}-\xi^{\mathrm{a}} \xi_{\mathrm{m}}\right) \xi^{\mathrm{n}} \nabla_{\mathrm{n}} \mathrm{v}^{\mathrm{m}}=\mathbf{0}$, as claimed.

9 If they are positioned so as to be orthogonal at some initial moment, they will remain so.

10 For example, the distance between them is constant as determined by the time it takes a light signal to complete a round trip passage from one particle to the other and back -- as measured by clocks sitting on the respective particles. Indeed, the distance between them is constant according to any notion of distance that can be formulated in terms of the spacetime metric $g_{a b}$ and the curves $\gamma_{X}$ and $\gamma_{Y}$, since they are all preserved under the flow maps associated with $\tau^{\mathrm{a}}$.

11 The proposition is slightly more general than the one proved in Rosquist (1980). He worked with a unit timelike vector field $\xi^{\mathrm{a}}$ that is Born rigid (i.e., has vanishing scalar expansion and shear) and geodesic. These two conditions imply that $\xi^{\mathrm{a}}$ is proportional to a Killing field, but not conversely. Rosquist also limited attention to the case where, in our notation, $v^{n} \omega_{n}=0$.

12 Every unit timelike field $\xi^{\mathrm{a}}$ whatsoever satisfies

$$
\nabla_{\mathrm{n}} \xi_{\mathrm{d}}=\theta_{\mathrm{nd}}+\omega_{\mathrm{nd}}+\xi_{\mathrm{n}} \xi^{\mathrm{m}} \nabla_{\mathrm{m}} \xi_{\mathrm{d}}
$$

where

$$
\theta_{\mathrm{nd}}=\mathrm{h}_{(\mathrm{n}}{ }^{\mathrm{r}} \mathrm{h}_{\mathrm{d})}{ }^{\mathrm{s}} \nabla_{\mathrm{r}} \xi_{\mathrm{s}} \quad \omega_{\mathrm{nd}}=\mathrm{h}_{[\mathrm{n}}{ }^{\mathrm{r}} \mathrm{h}_{\mathrm{d}]}{ }^{\mathrm{s}} \nabla_{\mathrm{r}} \xi_{\mathrm{s}} \quad \mathrm{h}_{\mathrm{nr}}=\mathrm{g}_{\mathrm{nr}}-\xi_{\mathrm{n}} \xi_{\mathrm{r}} .
$$

And every such field satisfies, $\varepsilon_{\text {ndpr }} \xi^{\mathrm{p}} \omega^{\mathrm{r}}=\omega_{\text {nd }}$ (as one can verify by direct 
substitution for $\omega^{\mathrm{r}}$ in the left side expression). But if $\tau \xi^{\mathrm{a}}$ is a Killing field for some scalar field $\tau, \theta_{\text {nd }}=\mathbf{0}$. (This follows from Killing's equation, $\nabla_{\mathrm{r}}\left(\tau \xi_{\mathrm{s}}\right)+\nabla_{\mathrm{s}}\left(\tau \xi_{\mathrm{r}}\right)=\mathbf{0}$.)

13 For an indication of what Gödel spacetime "looks like", see the diagrams in Hawking and Ellis (1973) and Malament (1984).

14 This is equivalent to the claim that $\tau^{\mathrm{n}} \nabla_{\mathrm{n}} \mathrm{r}=\mathbf{0}$ and $\tau^{\mathrm{n}} \nabla_{\mathrm{n}} \mathrm{y}=\mathbf{0}$. The first equation holds since $(\partial / \partial \mathrm{t})^{\mathrm{n}}(\partial / \partial \mathrm{r})_{\mathrm{n}}=0=(\partial / \partial \varphi)^{\mathrm{n}}(\partial / \partial \mathrm{r})_{n}$ and, hence,

$$
0=\tau^{\mathrm{n}}(\partial / \partial \mathrm{r})_{n}=\tau^{\mathrm{n}}\left(-4 \mu^{2}(\mathrm{dr})_{\mathrm{n}}\right)=-4 \mu^{2} \tau^{\mathrm{n}} \nabla_{\mathrm{n}} \mathrm{r} .
$$

The argument for the second equation is similar.

15 This picture, while helpful, is potentially misleading in one respect. As we shall see, a particle whose worldline is one of these helices can qualify as non-rotating relative to a particle whose worldline is an axis curve.

16 This fact explains the choice of the coefficient $\sqrt{2}$ in our expression for $\tau^{\mathrm{a}}$. We want $\omega^{\mathrm{a}}$ to be $\mathbf{0}$ at points where $\mathrm{r}=0$. As we show in the appendix, the vorticity associated with the general field $(\partial / \partial t)^{\mathrm{a}}+\mathrm{k}(\partial / \partial \varphi)^{\mathrm{a}}$ (where it is timelike) comes out to be

$$
\frac{\sqrt{2}+k\left(2 \operatorname{sh}^{2} r-1\right)+k^{2} \sqrt{2} \operatorname{sh}^{4} r}{\left(4 \mu^{2}\right)\left[1+k 2 \sqrt{2} \operatorname{sh}^{2} r+k^{2}\left(\operatorname{sh}^{4} r-s h^{2} r\right)\right]}(\partial / \partial y)^{a} .
$$

This reduces to

$$
\left(4 \mu^{2}\right)^{-1}(\sqrt{2}-\mathrm{k})(\partial / \partial \mathrm{y})^{\mathrm{a}} .
$$

at $\mathrm{r}=0$.

$17 \nabla_{(\mathrm{a}}(\partial / \partial \mathrm{y})_{\mathrm{b})}=\mathbf{0}$, since $(\partial / \partial \mathrm{y})^{\mathrm{a}}$ is a Killing field; and $\nabla_{[\mathrm{a}}(\partial / \partial \mathrm{y})_{\mathrm{b}]}=\mathbf{0}$, since 


$$
\nabla_{[\mathrm{a}}(\partial / \partial \mathrm{y})_{\mathrm{b}]}=-4 \mu^{2} \nabla_{[\mathrm{a}} \nabla_{\mathrm{b}]} \mathrm{y}=\mathbf{0}
$$

${ }^{18}$ We have

$$
\lambda^{\mathrm{n}} \nabla_{\mathrm{n}}\left(\lambda^{\mathrm{a}} \kappa_{\mathrm{a}}\right)=\lambda^{\mathrm{a}} \lambda^{\mathrm{n}} \nabla_{\mathrm{n}} \kappa_{\mathrm{a}}+\kappa_{\mathrm{a}} \lambda^{\mathrm{n}} \nabla_{\mathrm{n}} \lambda^{\mathrm{a}}=0
$$

The first term in the sum vanishes because $\kappa_{a}$ is a Killing field (and, so, $\nabla_{(n} \kappa_{a)}=0$ ). The second does so because $\lambda^{a}$ is a geodesic field.

19 See Lathrop and Teglas (1978) for an analytic characterization of geodesics passing through points where $r=0$, and see Hawking and Ellis (1973) for a picture.

20 The coordinate map t: $\mathrm{O} \rightarrow{ }^{\circledR}{ }^{4}$ qualifies as a global time function on $\left(\mathrm{O}, \mathrm{g}_{\mathrm{ab}} \mathrm{O}\right)$, i.e., it increases along all future-directed timelike curves. (Hence there cannot be any closed timelike curves in $\left(\mathrm{O}, \mathrm{g}_{\mathrm{ab}} \mid \mathrm{O}\right)$.) The assertion is equivalent to the claim that the vector field $\left(\nabla^{\mathrm{a}} \mathrm{t}\right)$ is timelike and future-directed (i.e., $\left(\nabla^{\mathrm{a}} \mathrm{t}\right)\left(\nabla_{\mathrm{a}} \mathrm{t}\right)>0$ and $\left.(\partial / \partial t)^{a}\left(\nabla_{a} t\right)>0\right)$ on $O$. But this is clear since

$$
\left(\nabla^{\mathrm{a}} \mathrm{t}\right)\left(\nabla_{\mathrm{a}} \mathrm{t}\right)=\frac{1-\operatorname{sh}^{2} \mathrm{r}}{4 \mu^{2}\left(1+\operatorname{sh}^{2} \mathrm{r}\right)} \text { on } \mathrm{O}
$$

and $(\partial / \partial t)^{a}\left(\nabla_{a} t\right)=1$ everywhere. (The expression for $\left(\nabla^{a} t\right)\left(\nabla_{a} t\right)$ follows from the fact that the inverse metric is:

$$
\begin{aligned}
\mathrm{g}^{\mathrm{ab}}= & \frac{1}{4 \mu^{2}\left(\operatorname{sh}^{2} \mathrm{r}+\operatorname{sh}^{4} \mathrm{r}\right)}\left[\left(\operatorname{sh}^{2} \mathrm{r}-\operatorname{sh}^{4} \mathrm{r}\right)(\partial / \partial \mathrm{t})^{\mathrm{a}}(\partial / \partial \mathrm{t})^{\mathrm{b}}-\left(\operatorname{sh}^{2} \mathrm{r}+\operatorname{sh}^{4} \mathrm{r}\right)(\partial / \partial \mathrm{r})^{\mathrm{a}}(\partial / \partial \mathrm{r})^{\mathrm{b}}\right. \\
& \left.\left.-\left(\operatorname{sh}^{2} \mathrm{r}+\operatorname{sh}^{4} \mathrm{r}\right)(\partial / \partial \mathrm{y})^{\mathrm{a}}(\partial / \partial \mathrm{y})^{\mathrm{b}}-(\partial / \partial \varphi)^{\mathrm{a}}(\partial / \partial \varphi)^{\mathrm{b}}+2 \sqrt{2} \operatorname{sh}^{2} \mathrm{r}(\partial / \partial \mathrm{t})^{(\mathrm{a}}(\partial / \partial \varphi)^{\mathrm{b})}\right] .\right)
\end{aligned}
$$

21 Let $\mathrm{R}$ be the closed set of points where $\mathrm{r}=0$. Since the fields $(\partial / \partial \mathrm{t})^{\mathrm{a}},(\partial / \partial \mathrm{r})^{\mathrm{a}}$, $(\partial / \partial \varphi)^{\mathrm{a}}$, and $(\partial / \partial \mathrm{y})^{\mathrm{a}}$ are linearly independent on $\mathrm{M}-\mathrm{R}$, there must exist some function $\mathrm{f}$ defined on $\mathrm{M}-\mathrm{R}$ for which the equation holds. We can determine $\mathrm{f}$, up 
to sign, with the following calculation:

$$
\begin{aligned}
&-(4 !)= \varepsilon^{\mathrm{abcd}} \varepsilon_{\mathrm{abcd}}=\mathrm{f}^{2}(\partial / \partial \mathrm{t})^{[\mathrm{a}}(\partial / \partial \mathrm{r})^{\mathrm{b}}(\partial / \partial \varphi)^{\mathrm{c}}(\partial / \partial \mathrm{y})^{\mathrm{d}]} \\
& \bullet(\partial / \partial \mathrm{t})_{[\mathrm{a}}(\partial / \partial \mathrm{r})_{\mathrm{b}}(\partial / \partial \varphi)_{\mathrm{c}}(\partial / \partial \mathrm{y})_{\mathrm{d}]} \\
&=\mathrm{f}^{2}(\partial / \partial \mathrm{t})^{[\mathrm{a}}(\partial / \partial \mathrm{r})^{\mathrm{b}}(\partial / \partial \varphi)^{\mathrm{c}}(\partial / \partial \mathrm{y})^{\mathrm{d}]} \\
& \qquad\left(4 \mu^{2}\right)^{4}\left(\left(\operatorname{sh}^{4} \mathrm{r}-\mathrm{sh}^{2} \mathrm{r}\right)-2 \mathrm{sh}^{4} \mathrm{r}\right)(\mathrm{dt})_{[\mathrm{a}}(\mathrm{dr})_{\mathrm{b}}(\mathrm{d} \varphi)_{\mathrm{c}}(\mathrm{dy})_{\mathrm{d}]} \\
&=-\mathrm{f}^{2}\left(4 \mu^{2}\right)^{4}\left(\mathrm{sh}^{4} \mathrm{r}+\mathrm{sh}^{2} \mathrm{r}\right) \frac{4 !}{(4 !)^{2}} .
\end{aligned}
$$

The volume elements on M-R defined by the two solutions for $f$ have well defined limits at points in R. Once those limit values are included, we have a (smooth) volume element on all of $\mathrm{M}$.

22 It is precisely here that the present argument would break down if we had not restricted attention to $\mathrm{O}$. 


\section{$\underline{\text { References }}$}

Hawking, S. W., and Ellis, G. F. R. (1973), The Large Scale Structure of Space-Time (Cambridge University Press, Cambridge).

Lathrop, J. and Teglas, R. (1978), "Dynamics in the Gödel Universe", Il Nuovo Cimento 43 B, 162-171.

Malament, D. (1986), "Time Travel in the Gödel Universe", PSA 1984, Volume 2 (proceedings of the Philosophy of Science Association meetings, 1984), 91-100.

Rosquist, K. (1980), "Global Rotation", General Relativity and Gravitation 12, 649664.

Wald, R. (1984), General Relativity (University of Chicago Press, Chicago). 


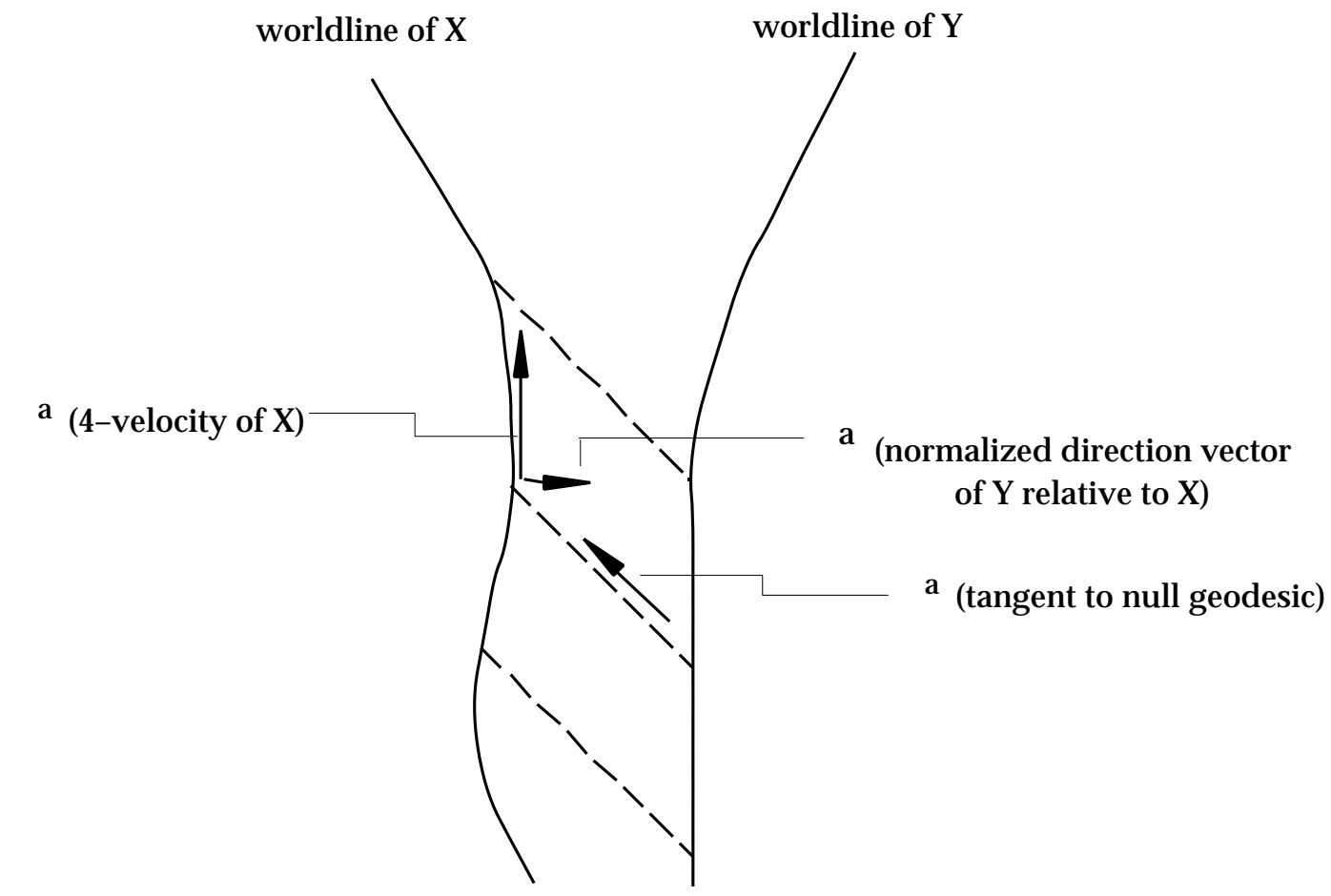

Figure 1 


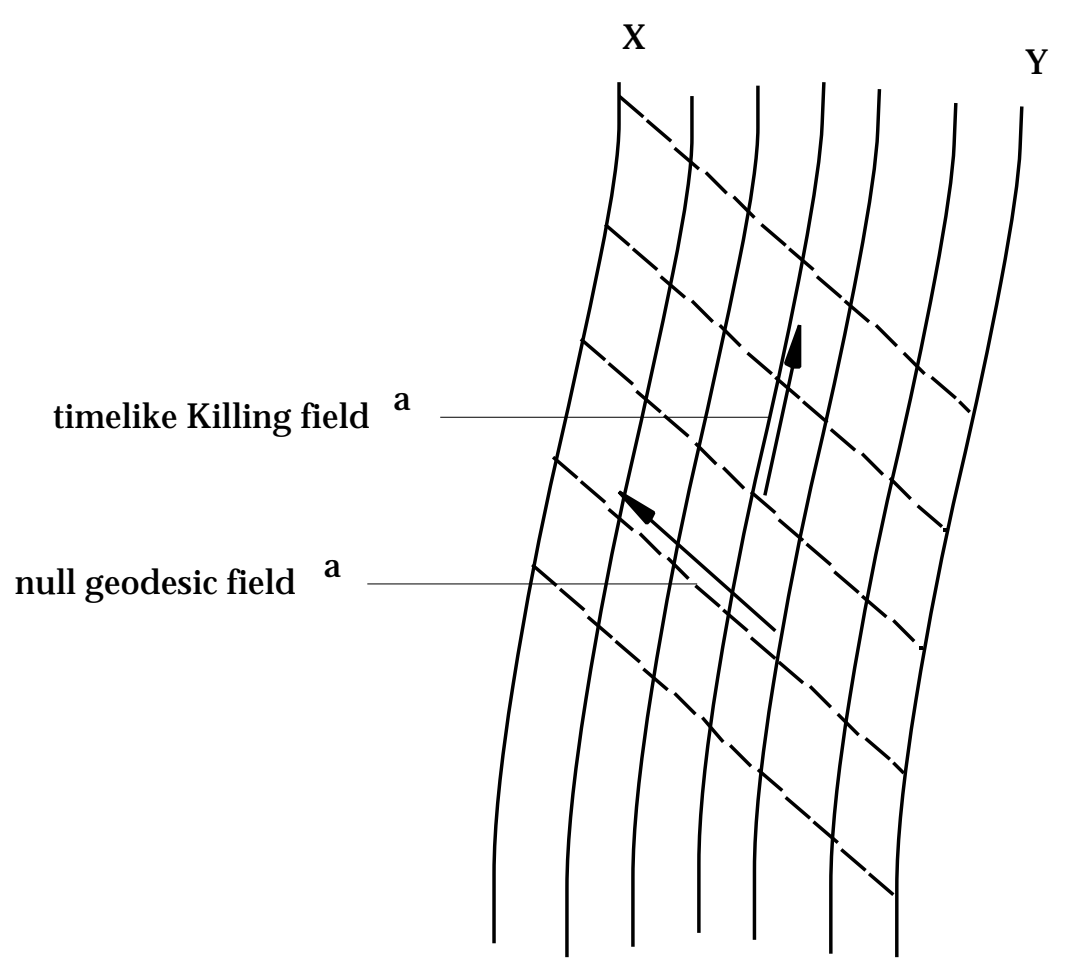

Figure 2 
$-27-$ 